\title{
Die Diffluenz des würmeiszeitlichen Rheingletschers bei Sargans (Kanton St. Gallen) und die spätglazialen Gletscherstände in der Walensee-Talung und im Rheintal ${ }^{1}$ )
}

\author{
Von René Hantke, Zürich
}

Mit 5 Abbildungen

A bstract. The formation of the bifurcated valley at Sargans (Eastern Switzerland) began in the Pliocene, during the emplacement of the Helvetic nappes. The Rhine Valley follows the megaflexure along the western boundary of the Austroalpine and Penninic nappes, the valley of Lake Walenstadt on the boundary between two Helvetic thrust sheets. Both valleys were deepened during Quaternary time. The divide in the valley of Lake Walenstadt was displaced successively to the southeast. In Late Wuerm time both valley bottoms reached nearly the same level.

Rhine erratics in the ablation area of the Linth glacier indicate that part of the Rhine ice flowed through the valley of Lake Walenstadt and joined the Linth glacier.

F. SAXER suggested that the Rhine ice joining the Linth system was insignificant, only $2-3 \%$, because a tributary of the Lake Walenstadt branch had dammed up the Rhine ice so that nearly its whole mass was forced to flow down the Rhine Valley. The occurrence of Rhine erratics $6 \mathrm{~km}$ up this tributary has proved that it was not able to dam up the Rhine ice; on the contrary, the equilibrium of ice masses was established far inside the tributary valley.

The Rhine glacier reached up to $1750 \mathrm{~m}$ above s. 1 . during the Wuerm maximum stage in the Sargans area. The percentage of Rhine ice flowing through the valley of Lake Walenstadt has been estimated by different methods; with results ranging from 28 to $40 \%$, the best value being $31 \%$.

According to the parameters given by Lliboutry or by Nye the mean rates of laminar flow varied from $50-150 \mathrm{~m} /$ year. These rates are much lower than the rates of ice movement deduced from annual snowfall which are $450-900 \mathrm{~m} /$ year. The difference can only be explained by assuming sliding movements, induced by meltwater films.

Meltwater channels can be observed at different levels in the Rhine Valley. On the Austrian side they connect with lateral moraines of Late Wuerm stages. The end moraines in the Feldkirch area show that the Ill glacier, a right tributary, readvanced again and almost joined a lateral tongue of the Rhine ice which had deposited an end moraine in front of them.

After some smooth stages of retreat in the Linth Plain, the combined Linth/Rhine glacier stopped at Ziegelbrücke. In a next phase the branch of Lake Walenstadt reached only its west end. Dam gravels at high levels on the mouths of tributaries, meltwater channels and ice-polished rocks bear evidence for these two stages of readvance and show that the local glaciers did not reach the main glacier.

Two still younger stages were recognized in the Sargans area by kame gravels and lateral meltwater channels at lower levels. Evidence for these stages can be observed far upstream the Rhine, in the Northern Grisons as well as at the junction of the Albula and the Rhine glaciers where beautiful end moraines enclose a small lateral basin. In the Domleschg area lateral moraines of local glaciers descended from the Staetzerhorn Range and nearly reached the valley bottom. These prove that there was a futher readvance, probably during Younger Dryas time.

$\mathrm{Z} u s$ a m me $\mathrm{n}$ a s sung. Auf Grund der Verteilung der höchsten Erratiker gelang es, die Eisoberfläche des würmzeitlichen Rheingletschers von Chur durch die Talgabelung von Sargans bis zur Konfluenz mit dem Linthgletscher einerseits und bis zur Einmündung des Illgletschers andererseits zu rekonstruieren.

Nach dem Koninuitätsprinzip ließen sich in ausgezeichneten Talquerschnitten Relativwerte der mittleren Fließgeschwindigkeit des Eises während des letzten Maximalstandes angeben. Mit den Parametern von LliboutRy bzw. von Nye wurde versucht, die Fließgeschwindigkeit in den entsprechenden Querschnitten größenordnungsmäßig zu berechnen. Gegenüber den Geschwindigkeiten, wie sie sich aus Wasserhaushalt-Überlegungen ergeben, sind sie jedoch viel zu gering, so daß

1) Vortrag anläßlich der 14. Tagung der Deutschen Quartärvereinigung in Biberach a. d. Riß am 6. 8. 1968. 
neben dem laminaren Fließen - besonders in den gefällsarmen Talabschnitten - mit einem erheblichen Gleiten auf der Unterlage zu rechnen ist.

In der Walensee-Talung wie im Rheintal konnten mehrere zeitlich sich entsprechende Rückzugshalte festgestellt werden. Einige markieren dabei deutliche spätglaziale Klimaverschlechterungen, da sie sich auch in einem kräftigeren Vorrücken der Seitengletscher abzeichnen. Durch Seitenmoränenreste lassen sich diese Stadien rheinaufwärts bis tief nach Bünden hinein verfolgen.

\section{Anlage der Talgabelung von Sargans}

Die Talgabelung von Sargans mit der Seez-Walensee-Talung und dem Rheintal ist auf tektonische Anlagen zurückzuführen. Bereits im Pliozän setzte mit der mise-en-place der Helvetischen Decken innerhalb des auseinander geglittenen Schichtstoßes die Bildung der Seez-Walensee-Talung ein. Längs der Großflexur zwischen den gegen E axial abtauchenden helvetischen Decken und dem westlichen Erosionsrand der penninischen und ostalpinen Decken tiefte sich das Rheintal ein (Abb. 1).

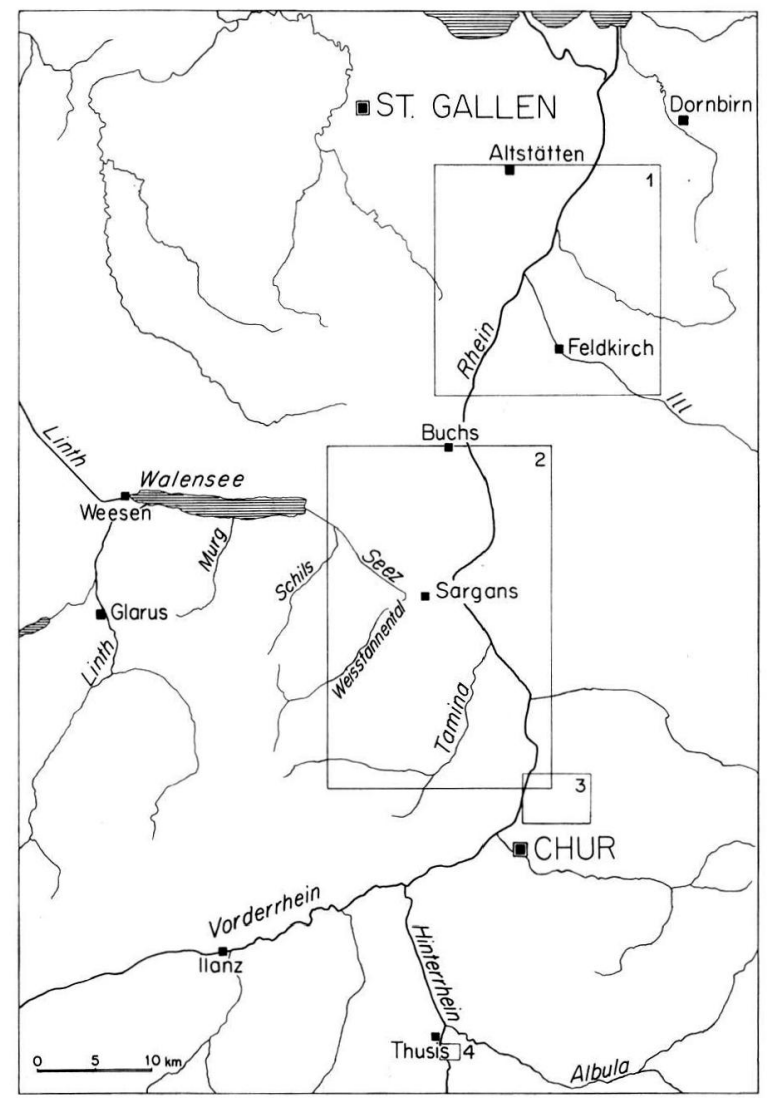

Abb. 1. Ubersichtsskizze der quartärgeologischen Kartenausschnitte.

Im Laufe des Quartärs rückte dann die Wasserscheide, die zwischen Walenseetal und Rheintal bestand und zunächst zwischen Prodchamm und Sichelchamm lag, schrittweise seeztalaufwärts; zugleich wurde sie vor allem durch die Glazialerosion immer stärker erniedrigt. Die heutige Gestalt der Talgabelung ist erst das Werk des würmeiszeitlichen Rheingletschers und holozäner Flußaufschüttungen. 
Rekonstruktion der Eisoberfläche des Rheingletschers

Auf Grund der höchsten Erratiker in Grat- und Terrassenanlagen im weiteren Bereich um Sargans ergab sich für den Rheingletscher an zahlreichen Stellen eine Mindest-Eishöhe zur Zeit des Würm-Maximums, so daß sich für dieses Gebiet eine Vergletscherungskarte zeichnen ließ.

Während die Eisoberfläche über Chur im Würm-Maximum in rund $2000 \mathrm{~m} \mathrm{lag,} \mathrm{sank}$ sie bis Sargans auf $1750 \mathrm{~m}$ ab. Wenig unterhalb Buchs, am N-Grat der Drei Schwestern, lag sie auf $1500 \mathrm{~m}$ und über Wildhaus, dem Transfluenzpaß ins Toggenburg, in $1480 \mathrm{~m}$. Im Talquerschnitt von Rüthi-Rankweil reichte das Rhein-Eis noch auf etwas über 1300 m. Die Karte von H. JäckцI (1962) gibt eine um bis $250 \mathrm{~m}$ höhere Eisgrenze wieder.

Eine damit gut übereinstimmende Eishöhe läßt sich in der Walensee-Talung im Durchbruch von Ziegelbrücke, unmittelbar nach dem Zusammentreffen von Walenseearm und Linthgletscher, ermitteln. Da auch die beiden Entfernungen Sargans-Ziegelbrücke und Sargans-Rüthi nahezu gleich groß sind, ergibt sich ein übereinstimmendes Gefälle der Eisoberflächen, und aus den gut miteinander vergleichbaren Eismächtigkeiten resultieren in beiden Gletscherarmen vergleichbare Fließgeschwindigkeiten. Einer allfälligen geringen Stauwirkung des Walenseearmes durch den Linthgletscher steht im Rheintal eine solche durch den Illgletscher gegenüber.

Während seit Arn. Escher (1852) auf Grund der Erratiker im Ablationsgebiet des Linthgletschers stets mit einem bedeutenden Abfluß von Rhein-Eis durch die WalenseeTalung gerechnet wurde, glaubt F. SAXER (1964), daß dieser Anteil 2-3\% nicht übersteige, da dieser durch den Weißtannengletscher gestaut worden wäre.

Daß jedoch beim Walenseearm ein nennenswerter Stau durch den aus dem Weißtannental abfließenden Seezgletscher ausblieb, geht aus der Tatsache hervor, daß das Rhein-Eis $6 \mathrm{~km}$ tief ins untere Weißtannental eindrang, dort Erratiker zurückließ und den Seezgletscher kräftig zurückstaute.

Fließgeschwindigkeiten eiszeitlicher Gletscher

Leider lassen sich Fließgeschwindigkeiten eiszeitlicher Gletscher höchstens abschätzen. Auf Grund des auch für fließendes Gletschereis gültigen Kontinuitätsprinzipes können aus dem Gefälle der Eisoberfläche gewisse Relativwerte angegeben werden. Wäre der Eisabfluß nach der Talgabelung von Sargans im Verhältnis der Gletscherquerschnitte aufgeteilt worden, so hätten rund $40 \%$ ihren Weg durch die Walensee-Talung und rund $60 \%$ durchs Rheintal genommen. Dabei wäre die mittlere Fließgeschwindigkeit nach der Diffluenz $\overline{\mathrm{v}}_{2}$ auf $0,83 \cdot \overline{\mathrm{v}}_{1}$ gefallen. Hätte sich diese, auf Grund der Trägheit des Eises des Hauptarmes, nicht geändert, so müßte der gesamte Geschwindigkeitsverlust den Walenseearm betroffen haben, wobei die Geschwindigkeit $\bar{v}_{3}$ auf $0,58 \cdot \bar{v}_{1}$ gesunken wäre. Danach wären noch immer $28 \%$ durch die Walensee-Talung abgeflossen. Im wahrscheinlichsten Fall, einer Verminderung der mittleren Fließgeschwindigkeiten im Verhältnis der Durchflußquerschnitte, ergäbe sich für den durch das Rheintal abfließenden Arm $\overline{\mathrm{v}}_{2}=$ $0,96 \cdot \bar{v}_{1}$ und für den seinen Weg durch die Walensee-Talung nehmenden Arm $\bar{v}_{3}=$ $0,64 \cdot \bar{v}_{1}$. Damit wäre der Eisabfluß zu gut $30 \%$ durch die Walensee-Talung und zu knapp $70 \%$ durch das Rheintal erfolgt.

Da nach J. F. Nye (1965) Fließgeschwindigkeiten von Gletschern mit der 3. Potenz ihres Gefälles ansteigen, ergibt sich für den Linthgletscher, der im untersten Linthtal ein Gefälle von $17 \%$ aufwies, eine mehr als doppelt so große laminare Fließgeschwindigkeit als im Walenseearm, der nur ein solches von 13,5\% \% erreichte.

Aus dem Kontinuitätsprinzip geht wiederum hervor, daß die Durchflußgeschwindigkeit im Durchbruch von Ziegelbrücke gegenüber derjenigen in der Walensee-Talung auf mehr als das Dreifache angestiegen sein muß, während sie in der Linthebene, wo sich das Eis wieder ausbreiten konnte, im umgekehrten Verhältnis zur Querschnittsvergrößerung wieder abnahm. 
Durchflußmengen, Durchflußgeschwindigkeiten, mittlerer laminarer Durchfluß und mittlere laminare Fließgeschwindigkeiten ergeben nach Lliboutry (1965) bzw. Nye (1953) in den einzelnen Talquerschnitten des Rhein- und des Linthgletschers folgende Werte:

\begin{tabular}{|c|c|c|c|c|c|c|}
\hline \multirow[t]{2}{*}{ Gletscherquerschnitt } & \multicolumn{2}{|c|}{$\begin{array}{l}\text { jährlicher laminarer } \\
\text { Durchfluß in } \mathrm{m}^{3}\end{array}$} & \multirow{2}{*}{$\begin{array}{l}\text { Gesamt- } \\
\text { durchfluß } \\
\text { in } \mathrm{m}^{3} / \mathrm{Jahr} \\
\text { Massen- } \\
\text { haushalt }\end{array}$} & \multicolumn{2}{|c|}{$\begin{array}{l}\text { laminare } \\
\text { Fließgeschw. } \\
\text { in } \mathrm{m} / \mathrm{Jahr}\end{array}$} & \multirow{2}{*}{$\begin{array}{l}\text { Durchfl.- } \\
\text { geschw. } \\
\text { in m/Jahr } \\
\text { Massen- } \\
\text { haushalt }\end{array}$} \\
\hline & $\begin{array}{c}\text { nach } \\
\text { LLIBOuTRY }\end{array}$ & $\begin{array}{l}\text { nach } \\
\text { NyE }\end{array}$ & & $\begin{array}{l}\text { nach } \\
\text { LLIB. }\end{array}$ & $\begin{array}{l}\text { nach } \\
\text { NyE }\end{array}$ & \\
\hline Rheingletscher oberhalb Sargans & $4,6 \cdot 10^{8}$ & $10,4 \cdot 10^{8}$ & $72,7 \cdot 10^{8}$ & 45 & 100 & 700 \\
\hline Rheingletscher unterhalb Sargans & $5,7 \cdot 10^{8}$ & $12,6 \cdot 10^{8}$ & $50,3 \cdot 10^{8}$ & 75 & 168 & 672 \\
\hline Walenseearm unterhalb Sargans & $3,2 \cdot 10^{8}$ & $7,3 \cdot 10^{8}$ & $22,4 \cdot 10^{8}$ & 65 & 145 & 448 \\
\hline Walenseearm oberhalb Ziegelbrücke & $1,5 \cdot 10^{8}$ & $3,4 \cdot 10^{8}$ & $30,7 \cdot 10^{8}$ & 46 & 100 & 902 \\
\hline Linthgletscher oberhalb Ziegelbrücke & $2,6 \cdot 10^{8}$ & $5,8 \cdot 10^{8}$ & $13,6 \cdot 10^{8}$ & 86 & 192 & 453 \\
\hline Linth/Rheingletscher unterhalb Ziegelbrücke & $10,8 \cdot 10^{8}$ & $24,0 \cdot 10^{8}$ & $44,3 \cdot 10^{8}$ & 357 & 800 & 1477 \\
\hline
\end{tabular}

Auf Grund des Massenhaushaltes in beiden Gletschersystemen resultieren jedoch - unter Annahme von 10\% höheren Niederschlagsmengen, einem Verdunstungsanteil und einem subglazialen Abfluß von je 10\% - wesentlich größerer Durchflußgeschwindigkeiten. Diese Diskrepanz läßt - trotz der mangelhaften Kenntnisse und der vom parabolischen Querschnitt oft stark abweichenden Talgestalt - nur den Schluß zu, daß auch bei der Bewegung eiszeitlicher Gletscher neben der laminaren Fließgeschwindigkeit ein erheblicher Anteil auf Gleitvorgänge zurückzuführen ist. Dies gilt besonders für die gefällsarmen Talabschnitte.

Eisstände, die den ersten hochwürmeiszeitlichen Rückzugsstadien zuzuordnen sind, lassen sich im weit über der Schneegrenze gelegenen Bereich der Talgabelung von Sargans nicht mit Sicherheit feststellen. Wohl stellen sich ab und zu kleinere terrassenartige Verflachungen ein; ebenso scheinen Erratiker zuweilen höhenschichtmäßig gehäuft.

Die spätw ürmzeitlichen Gletscherstände in beiden Talungen

Auf das Hurden-(Konstanzer-)Stadium folgen im Walenseearm wie im Hauptarm des Rheingletschers verschiedene Rückzugshalte, die sich, wenn auch nicht durch Stirnmoränen, so doch durch Wallmoränenreste, seitliche Schmelzwasserrinnen, Stauschotterfluren, Rundhöcker und teilweise bereits wieder eingeschotterte und verlandete Zungenbecken abzeichnen.

Beim Linth/Rhein- und beim Bodensee-Rheingletscher lassen sich über die Diffluenz von Sargans hinaus folgende, zeitlich einander entsprechende spätwürmzeitliche Eisstände unterscheiden:

\begin{tabular}{l|l}
\hline Linth/Rheingletscher & Bodensee-Rheingletscher \\
\hline Hurden - R a p p erswil & Konstan z \\
Schübelbach - Schmerikon & Heerbrugg - Dornbirn \\
Buttikon - Uznach & Rebstein - Widnau - Hohenems \\
Reichenburg - Maseltrangen & Altstätten - Altach \\
Bilten - Schänis & Kobelwald - Montlingen - Götzis \\
Ziegelbrücke & Gruppen - Rüthi - Hirschensprung - Rankweil \\
We esen & Sennwald - Büchel - Fe ld kirch \\
Ragnatsch & Wartau - B a l ze r s \\
Mels & Sarganser Au \\
\hline Gesperrt: Endmoränenreste. &
\end{tabular}




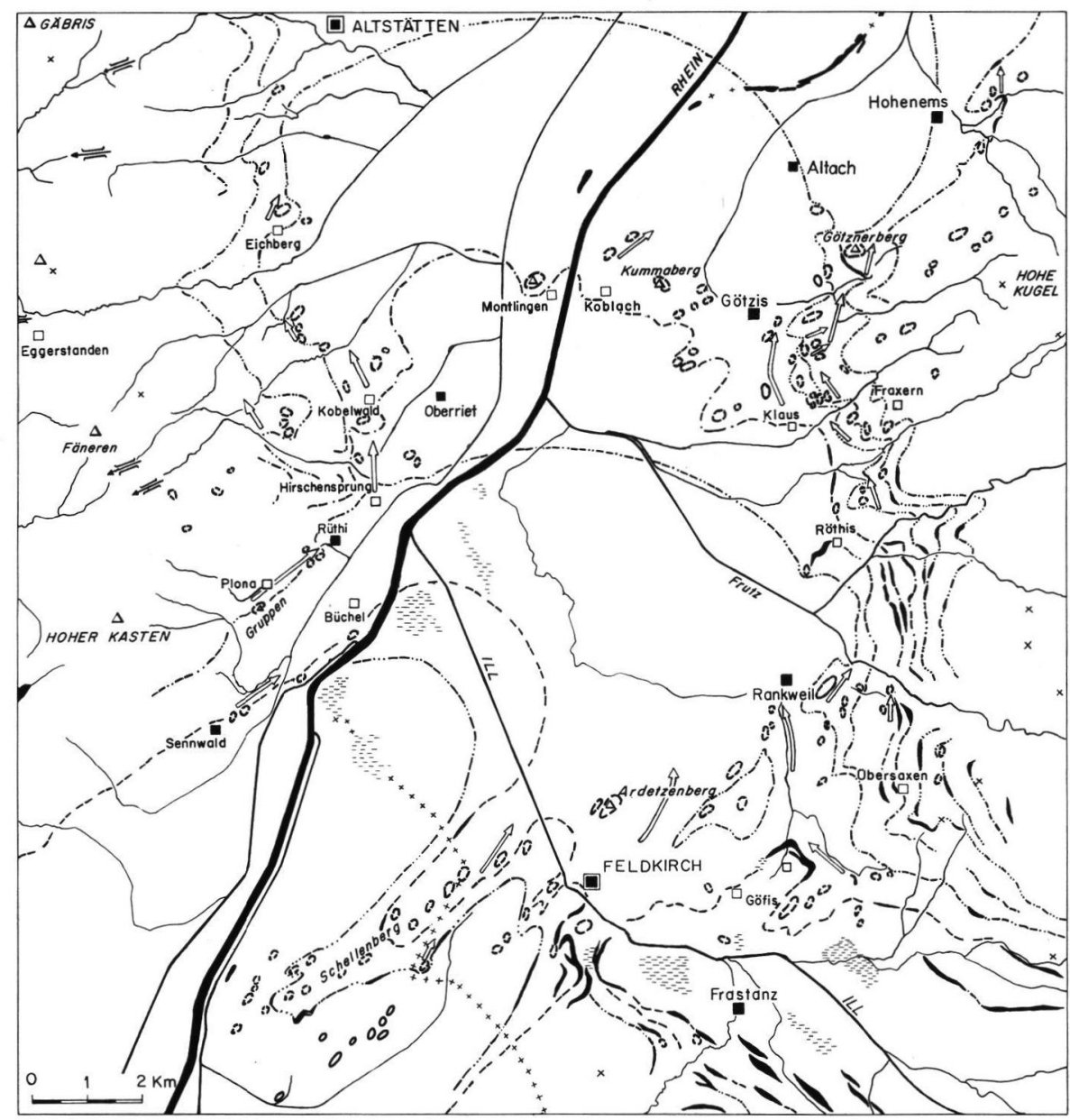

Abb. 2. Quartärgeologische Kartenskizze des Rheintales zwischen Feldkirch und Altstätten.

\begin{tabular}{|c|c|}
\hline$x x^{x}$ & $\begin{array}{l}\text { Höchste würmeiszeitliche } \\
\text { Erratiker }\end{array}$ \\
\hline$=$ & Versumpfte Zungenbecken \\
\hline 000 & Drumlins \\
\hline 30 & Rundhöcker \\
\hline & Moränenwälle \\
\hline$=$ & Schmelzwasserrinnen \\
\hline & Eistransfluenzen \\
\hline & Spätglaziale Schotter \\
\hline & Melser Staffel \\
\hline & $\begin{array}{l}\text { Plonser Staffel } \\
\text { Eisstände von Ziegelbrücke } \\
\text { bzw. Weesen }\end{array}$ \\
\hline
\end{tabular}

Eisstände

\begin{tabular}{|c|c|}
\hline & Walensee-Talung \\
\hline$-\cdot-\cdot-\cdot-\cdot \cdot \cdot-$ & \\
\hline$-\cdots-\cdots-\cdots-\cdots-\cdot$ & Buttikon - Uznach \\
\hline$-\cdots-\cdots-\cdots-\cdots-$ & \\
\hline$--\cdot--\cdot--\cdot-$ & Reichenburg - Maseltrangen \\
\hline$-\cdots-\cdots-\cdots--\cdots$ & Ziegelbrücke \\
\hline$--\quad-----$ & Weesen \\
\hline--------- & $\begin{array}{l}\text { Ragnatsch } \\
\text { Plons }\end{array}$ \\
\hline$\ldots \ldots \ldots \ldots \ldots \ldots \ldots \ldots$ & Mels \\
\hline
\end{tabular}

Rheintal

Heerbrugg - Dornbirn

Widnau - Hohenems

Altstätten - Altach

Montlingen - Götzis

Rüthi - Rankweil

Büchel - Feldkirch

Sennwald - Tisis

Wartau

Azmoos

Sarganser Au 


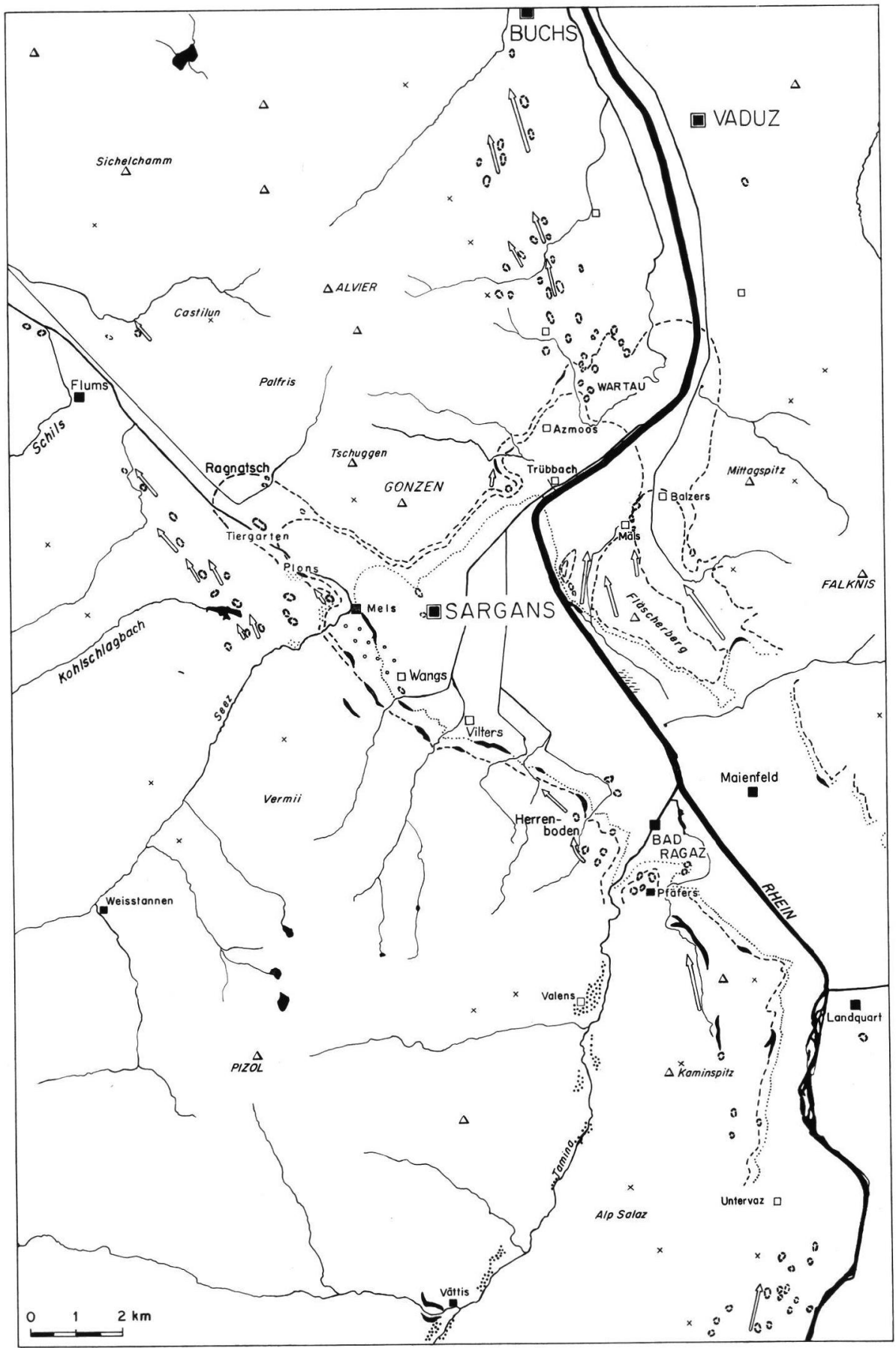

Abb. 3. Quartärgeologische Kartenskizze der weiteren Umgebung der Talgabelung von Sargans. 


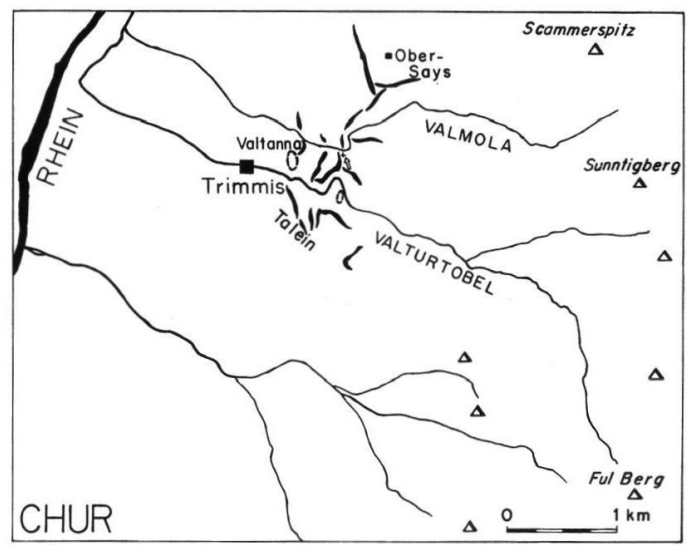

Abb. 4. Quartärgeologische Kartenskizze der Umgebung von Trimmis NE von Chur.

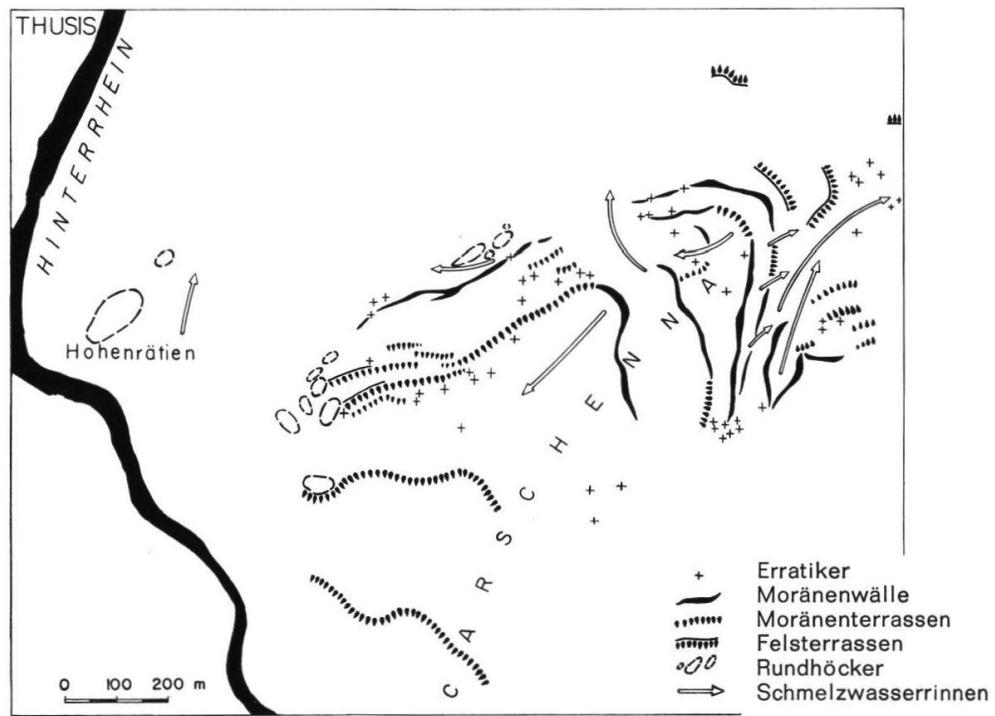

Abb. 5. Quartärgeologische Kartenskizze von Carschenna SE von Thusis.

Die Tatsache, daß in Vorarlberg mehrere randliche Schmelzwasserrinnen - etwa um Götzis, Rankweil und Feldkirch - klar in Erscheinung treten und sich stellenweise mit gut ausgebildeten Seitenmoränen verbinden lassen, deutet darauf hin, daß der Rheingletscher noch im Spätwürm mehrmals leicht vorrückte und dann jeweils eine gewisse Zeit stagnierte, so daß den Schmelzwässern genügend Zeit verblieb, Abflußrinnen auszukolken (Abb. 2).

Eine Seitenmoräne, die dem Stadium von Feldkirch/Weesen zuzuordnen ist, konnte im Churer Rheintal bei Ober Says in $1100 \mathrm{~m}$ erkannt werden (Abb. 4). Zeitlich den Stadien um Sargans (Abb. 3) entsprechende Eisstände finden sich ebenfalls oberhalb Trimmis in $900 \mathrm{~m}$ (Abb. 4). Sie lassen sich noch E von Thusis als scharf ausgebildete Moränenwälle des Hinterrheingletschers im Konfluenzbereich mit dem Albulagletscher beobachten (Abb. 5). 
Da auch die Seitengletscher - Murg-, Schils-, Weißtannen- und Taminagletscher nach einer ersten Abschmelzphase nochmals deutlich vorstießen, drückt sich in diesen spätglazialen Stadien eine kurzfristige allgemeine Klimaverschlechterung mit mehreren kleineren Kältespitzen aus. Dabei fällt auf, daß nur Gletscher aus steilen Seitentälern den Hauptgletscher noch erreichten, während beim Weißtannen- und beim Taminagletscher an den Talausgängen mächtige Schottermassen aufgestaut wurden (Abb. 3). Obwohl die zeitliche Einstufung dieser späteiszeitlichen Stadien bisher pollenanalytisch noch nicht vorgenommen werden konnte, dürfte sie wahrscheinlich der Älteren Dryaszeit entsprechen, die auf die erste kräftigere Erwärmung der ausgehenden Würmeiszeit, auf das BöllingInterstadial, folgte.

Im Domleschg zeichnet sich in Form eines bedeutenden Vorstoßes der Seitengletscher von der Stätzerhorn-Kette ein zweiter fühlbarer Kälterückschlag ab. Anzeichen eines Zungenbeckens, einer zugehörigen Sanderflur sowie tiefliegende Rundhöcker und randliche Schmelzwasserrinnen sprechen dafür, daß damals der Hinterrheingletscher - unterstützt durch mehrere Seitengletscher - abermals vorstieß und nochmals die Talsohle des Domleschg erfüllte. Eine mächtige Flur schlecht gerundeter, eisrandnaher Schotter wurde durch die Bergsturzmassen von Reichenau aufgestaut. Da diese am ehesten in einem vorangegangenen zweiten Interstadial - wahrscheinlich im Alleröd - niederbrachen, denn erst damals gaben die Talgletscher in Bünden die übersteilten Flanken erstmals frei, erfolgte der zweite Gletschervorstoß wahrscheinlich in der Jüngeren Dryaszeit.

Für den Hinweis von Berechnungsmöglichkeiten der laminaren Fließgeschwindigkeiten und der jährlichen Durchflußmengen auf Grund des Massenhaushaltes bin ich Herrn Dr. H. RöthlisBERGER von der Abteilung für Hydrologie und Glaziologie der ETH zu Dank verpflichtet.

Die Reinzeichnung der Kartenskizzen besorgte Herr U. MasonI.

Eine ausführlichere Arbeit mit einer Vergletscherungskarte des Raumes zwischen Ringelspitzkette und Alpstein erscheint in der Vierteljahrsschrift der Naturforschenden Gesellschaft in Zürich, 113.

\section{Literatur}

Escher, Arn.: Über die Bildungsweise der Landzunge von Hurden im Zürichsee. Mitth. naturf. Ges. Zürich 2, Nr. 71 und 72, 506-515, Zürich 1852.

JäckLI, H.: Karte der letzten Vergletscherung (Würmeiszeit) der Schweiz 1:1000000. In: DE Quervain, F. et al.: Geotechn. Karte Schweiz 1:200 000, Bl. 2, 2. Aufl. und Eclogae geol. Helv. 55/2, Taf. 1. Basel 1962.

NyE, J. F.: The flow of ice from measurements in glacier tunnels, laboratory experiments and the Jungfraufirn borehole experiment. Proc. Roy. Soc., A, 219, 477-489, London 1953. - - The flow of glacier in a channel of rectangular, elliptic or parabolic cross-section. J. Glaciol. 5, 661-690. London 1965.

SAXER, F.: Die Diffluenz des Rheingletschers bei Sargans. Eclogae geol. Helv. 57/2, 604-607. Basel 1964.

Manuskr. eingeg. 28. 8. 1968.

Anschrift des Verf.: Prof. Dr. R. Hantke, 8006 Zürich, Geologisches Institut der Eidg. Technischen Hochschule, Sonneggstraße 5. 